\title{
Maximizing the Information Content in Electron Microscopy Images
}

Raphael Marchand ${ }^{1,2}$, Thomas Juffmann ${ }^{1,2}$

${ }^{1}$ University of Vienna, Faculty of Physics, VCQ, A-1090 Vienna, Austria.

${ }^{2}$ University of Vienna, Max Perutz Laboratories, Department of Structural and Computational Biology, A-1030 Vienna, Austria.

Cryogenic electron microscopy and tomography have led to a revolution in structural biology, enabling the reconstruction of proteins, and sub-cellular structure, respectively. However, dose induced damage and shot noise limit the signal-to-noise that is achievable with these techniques. It prevents us from reconstructing the atomic structure of a single protein, and from identifying small proteins with masses below approximately $50 \mathrm{kDa}[1]$.

Imaging biological specimens is often done using phase-sensitive techniques, as low Z materials mostly affect the phase of the electron wave function, and not its amplitude. Here, we discuss the information content in phase microscopy images, as well as two recent proposals to overcome current limits in sensitivity.

The information content in noisy measurements can be quantified using the concept of Fisher information, which has recently been done for optical phase microscopy [2,3]. The results can be applied to electron microscopy and allow comparing common electron microscopy schemes such as phase contrast microscopy (using a phase plate) and off-axis holography. The analysis based on Fisher information also allows calculating the respective Cramer-Rao bounds, which give a lower bound on the phase estimation errors that can be achieved. Phase contrast microscopy yields optimal results for thin specimens, and for the case of plane wave illumination. Achieving even lower phase estimation errors requires unconventional approaches that either change the incoming state of the electrons, or the way the information is encoded onto the electron state.

Two ways to change the way the information is encoded onto the electron state are multi-pass transmission electron microscopy (MPTEM) [4,5] and Optical Near-field Electron Microscopy (ONEM)[8]: In MPTEM, each electron traverses the sample multiple times, which increases the achievable signal to noise per electron-sample interaction. Both Fisher information and measurement precision thus increase at constant damage. Image simulations show that MPTEM could enable the imaging of the secondary structure of single proteins.

Recently, ONEM has been proposed as a way to circumvent electron-induced damage [6]: In ONEM, a sample is probed non-invasively using light, and the resulting near-field interference patterns are converted into an electron current using a photocathode. The emitted electrons are then imaged with nanometric resolution using a modified Low Energy Electron Microscope (LEEM) [7]. Figure 1 shows an image of two gold nanospheres (diameter $5 \mathrm{~nm}$ ) simulated with the MNPBEM method [8]. The two particles are transversally separated by a $10 \mathrm{~nm}$ distance, and at different distances to the photocathode. One can see that ONEM in principle offers nanometric resolution, which is approximately given by the sample-photocathode distance. For sample-photocathode distances much smaller than the optical wavelength $(z \ll \lambda)$ the image contrast decreases drastically with distance $\left(\propto 1 / z^{3}\right)$. This allows for excellent suppression of background signals. ONEM thus represents a new scheme for low-damage, high- 
resolution imaging of interfaces.

References:

[1] RM Glaeser, Nat. Meth. 13 (2016), p. 28.

[2] D Bouchet et al, Physical Review Applied 15 (2021), p. 024047.

[3] S Koppell and M Kasevich, Optica 8 (2021), p. 493.

[4] T Juffmann et al, Scientific Reports 7 (2017), p. 1699.

[5] SA Koppell et al, Ultramicroscopy 207 (2019), p. 112834.

[6] R Marchand et al, Physical Review Applied 16 (2021), p. 12102.

[7] D Geelen et al, Review Letters 123 (2019).

[8] U Hohenester and A Trügler, Computer Physics Communications 183 (2012), p. 370.

a)

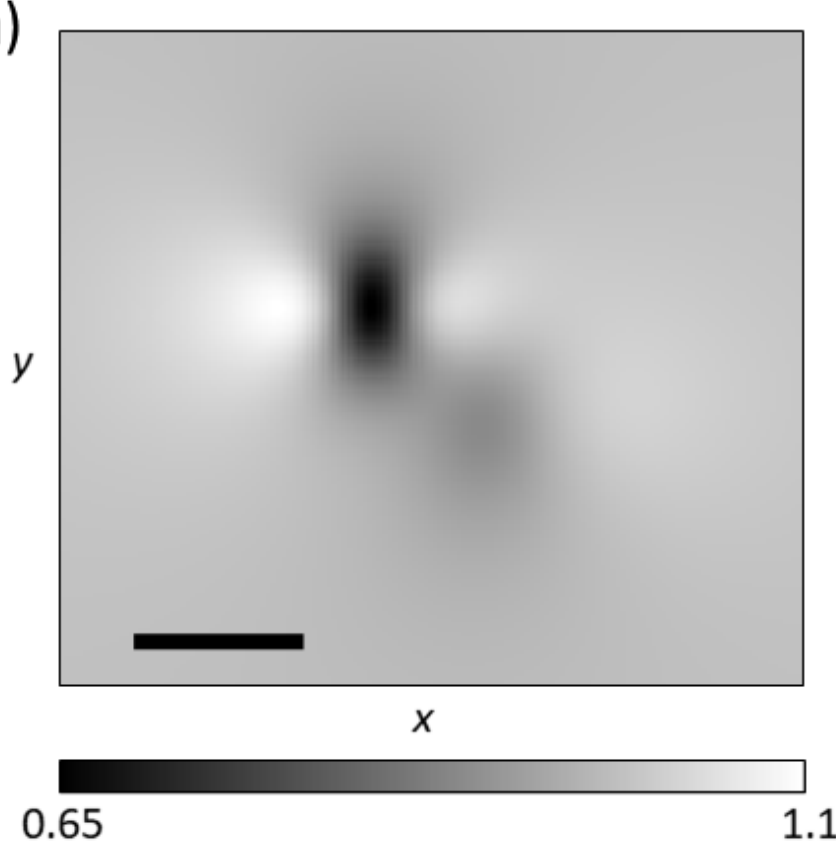

b)

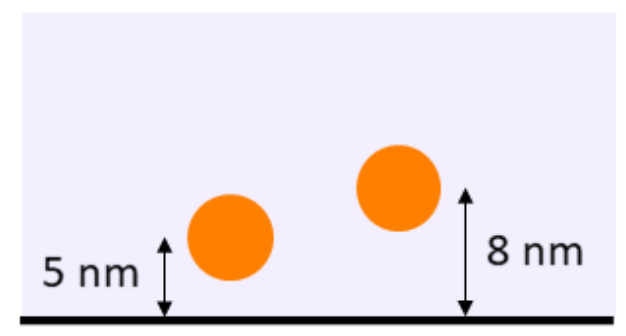

water gold photocathode

Figure 1. a) Simulated ONEM image of two gold nanoparticles (diameter $5 \mathrm{~nm}$ ), in water, next to each other, at a distance $\mathrm{z}=5 \mathrm{~nm}$ (top left) and $\mathrm{z}=8 \mathrm{~nm}$ (bottom right) from the photocathode, using the MNPBEM method. The grayscale shows the intensity normalized to the intensity of the excitation field. The excitation field is polarized along the $\mathrm{x}$ direction. The contrast and FWHM of the simulated near-field intensity distributions are $62 \%$ and $4.0 \mathrm{~nm}$ for the particle at $\mathrm{z}=5 \mathrm{~nm}$ and $16 \%$ and $6.8 \mathrm{~nm}$ for the particle at $\mathrm{z}=8 \mathrm{~nm}$ from the photocathode. The scale bar is $10 \mathrm{~nm}$. b) Schematic cross section of the 2 gold nanoparticles arrangement used for the simulation of a). The distance to the photocathode is indicated with the arrows. The photocathode is assumed to be infinitely thin. The particles are immersed in water. 